Hydraulic Engineering Repository

Ein Service der Bundesanstalt für Wasserbau

Kopmann, Rebekka; Merkel, Uwe H. Drei Jahre Telemac Open Source

Verfügbar unter / Available at:

https://hdl.handle.net/20.500.11970/100688

Vorgeschlagene Zitierweise / Suggested citation:

Kopmann, Rebekka; Merkel, Uwe H. (2013): Drei Jahre Telemac Open Source. In: Wasserwirtschaft Jg. 103 (12/2013). S. 10-15. 
Erstveröffentlichung in WasserWirtschaft 12 (2013), S. 10-15.

Für eine korrekte Zitierbarkeit ist die Seitennummerierung der Originalveröffentlichung für jede Seite kenntlich gemacht.

\section{Drei Jahre Telemac Open Source}

\section{Rebekka Kopmann und Uwe H. Merkel}

Der Übergang zu quelloffener Software stellt einen Paradigmenwechsel für numerische Software im Bereich der Fließgewässerhydraulik dar. Durch die gemeinschaftlich basierter Entwicklung von großen Softwarepaketen, wie der Telemac-Suite, entsteht eine starke Dynamik: die Nutzerzahlen steigen deutlich an und mit ihnen entstehen viele Ideen, Neuentwicklungen und Projekte. Das Telemac-Konsortium koordiniert und gewährleistet Kontinuität und Qualität. Dieser Artikel gibt einen Überblick, wie Ingenieure in Planungsbüros, Behörden und Forschung davon profitieren können.

\section{Einleitung}

Die hydraulische Modellierung von Fließgewässern erlebt in den letzten Jahren tief greifende strukturelle Veränderungen. Während vor wenigen Jahren fast jede Hochschule und zahlreiche Firmen eigene Programmpakete entwickelt haben, bündeln mittlerweile viele Förderer der Branche ihre Kräfte, auch über Landesgrenzen hinweg.

Drei Vorteile haben diese Entwicklung maßgeblich befördert und die Software-Eigentümer dazu bewogen auf Lizenzeinnahmen zu verzichten sowie den Quellcode frei zugeben:

- Zuerst sind natürlich der relativ übersichtlicheAnwenderkreis und die immerwied er individuellen Aufgaben zu nennen. Eine Software mit einem allumfassenden Funktionsumfang kann aus Kostengründen niemand bieten. Aber eigene projektspezifische Erweiterungen bereits validierter Basismodule kann an einer quell-offenen Software jeder vornehmen, der die nötigen Kenntnisse in der Softwareentwicklung mitbringt.

- Wie bei fast allen ingenieurspezifischen und wissenschaftlichen Softwareentwicklungen ist das wichtigste die Akzeptanz des Verfahrens durch Auftraggeber, aber auch durch Kritiker. Akzeptanz wird durch Transparenz erreicht. Je größer der Benutzerkreis ist, desto schneller fallen Ungereimtheiten im Verfahren auf.

- Kaum einem Unternehmen ist es allein möglich, den Funktionsumfang und den Benutzersupport für ein solches Produkt zu bieten. Die Beiträge von Universitäten, von Mitgliedern in Internetforen und Drittentwicklern sorgen für einen erheblichen Mehrwert.

Telemac ist einer der am weit verbreitetsten Vertreter der neuen Generation quell-offener Software-Pakete zur Berechnung von Strömungen mit freier Oberfläche. Bereits vor knapp 27 Jahren begann das Wasserbaulaboratorium der Electricité de France (EDF) mit der Entwicklung von Telemac. 


\section{Autorenfassung}

Kopmann, Merkel: Drei Jahre Telemac Open Source, 2013

Mit Anwendungen in allen Bereichen rund um Oberflächengewässer bietet die Telemac-Suite ein mächtiges Werkzeug für die Strömungssimulation: Es können 2-D- und 3-D-Strömungen in Binnengewässern und Meeren simuliert werden. Wind, Wellen sowie Geschiebe- und Schwebstofftransport können autark oder gekoppelt berechnet werden. Ein Grundwassermodell für zwei bzw. drei Dimensionen ist ebenfalls integriert, ist allerdings in der quelloffenen Software-Version noch nicht verfügbar. Die Telemac-Suite kann auf den Betriebssystemen Windows, Linux oder Unix installiert werden und ist in der Lage, parallele Architekturen effizient zu nutzen, egal ob auf Laptop, Workstation, Cluster und bei kurzfristig hohem Arbeitsaufkommen auch in der gemieteten HighPerformance-Computing-Cloud (HPC-Cloud).

Seit Freigabe der Quellen im Jahr 2010 ist das Programm kostenlos erhältlich und wird maßgeblich von einem Konsortium aus sechs Kernanwendern in Frankreich, Großbritannien und Deutschland weiterentwickelt sowie validiert. Zu diesem Konsortium gehören Artelia (ehemals Sogreah, F), die Bundesanstalt für Wasserbau (BAW, D), Centre d'Etudes Techniques Maritimes et Fluviales (CETMEF, F), Daresbury Laboratory (UK), Electricité de France R\&D (EDF, F) und HR Wallingford (UK). Beiträge von zahlreichen Drittentwicklern werden ebenfalls geprüft und eingepflegt.

Validierungen, wie z. B. das von Goll und Kopmann [1] vorgestellte Projekt, Weiterentwicklung des Funktionsumfangs und eine kontinuierlicheVerbesserung der Prognosefähigkeit sind kosten- und zeitintensiv, aber unbedingt notwendig. EinzelneEntwickler können diesen Aufwand nicht betreiben. Durch das Open-Source-Konzept sind die daraus entstandenen Verbesserungen frei zugänglich und das öffentliche Fachpublikum profitiertvon diesen Arbeiten. Als Gegenleistung sind von Seiten der Entwickler ausdrücklich Rückmeldungen der Benutzergemeinde zur weiteren Verbesserung erwünscht.

Die Beiträge in dieser Ausgabe der Fachzeitschrift WasserWirtschaft sollen einen Überblick über den Stand der Möglichkeiten der Telemac-Suitegeben und die Wissenschaftler sowie Ingenieure im deutschsprachigen Raum animieren, die Telemac-Suite zu testen, zu nutzen und zu ihrer Entwicklung beizutragen.

Dabei stehen sowohl die praktische Anwendung als auch die wissenschaftlichen Grundlagen im Fokus, die Softwarearchitektur wird nur bei Bedarf kurz angerissen. Aktuelle Anwendungen aus verschiedenen Bereichen des Wasserbaus in Behörden, Unternehmen und der Forschung zeigen das weite Anwendungsspektrum und die fundierte Grundlage der Telemac-Suite.

\section{Telemac-Module und -Neuentwicklungen}

Zum allgemeinen Grundverständnis wird in diesem Artikel ein kurzer Überblick 


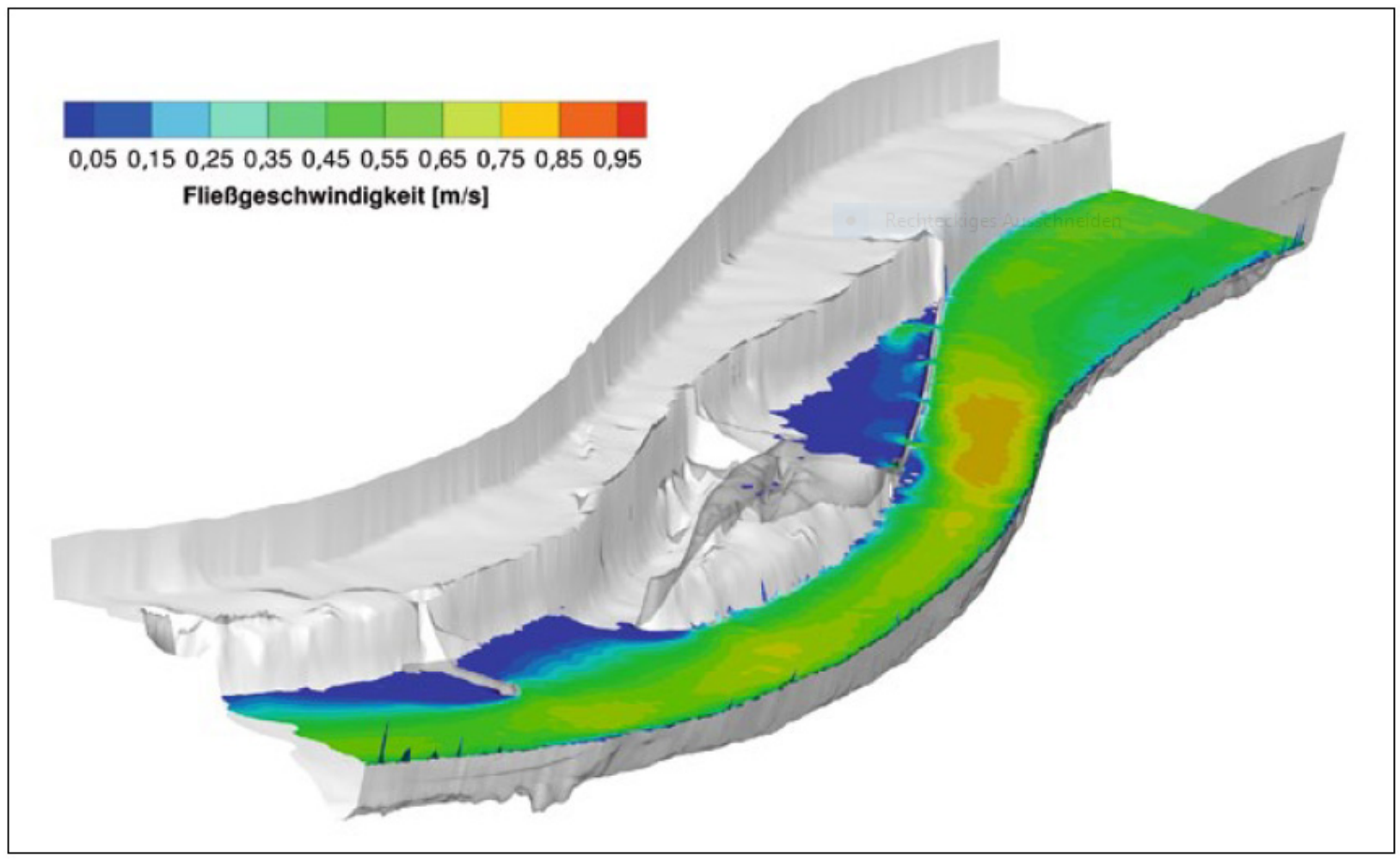

Bild 1: $\quad$ Mit Telemac-2D berechnete tiefengemittelte Fließgeschwindigkeit für das Validierungsbeispiel „221_Donau“

über die sechs Module der Telemac-Suite Telemac-2D, Telemac-3D, Sisyphe, DredgeSim, Tomawac und Artemis gegeben.Zudem werden einige der jüngsten Entwicklungen für den Bereich Sedimenttransport vorgestellt. Weitere Informationen finden sich unter www.opentelemac. org und bei [2].

\subsection{Hydrodynamik (Telemac-2D und Telemac-3D)}

Hydrodynamische2-D-Berechnungen von Flüssen und Ästuaren gehören mittlerweile zum Stand der Technik. Typische Anwendungen für Telemac-2D aus der Praxis umfassen z. B. Überflutungsszenarien, Beeinflussung der Strömungssituation mittels Wasserbauwerken oder Schubspannungsanalysen. Eine besondere Stärke von Telemac-2D und auch Telemac-3D sind die Wahlmöglichkeiten verschiedener Teilmodule. In Telemac-2D kann z. B. zwischen 7 Rauheitsgesetzen und 4 Turbulenzmodellen gewählt werden. Daneben sind viele für die Praxis relevante Randbedingungen, z. B. Wasserstand-Abflusskurven, vorgesehen. Auch im numerischen Bereich gibt es viele Möglichkeiten, z. B. kann zwischen Finite Elementen oder Finite Volumen entschieden wer- 


\section{Autorenfassung}

Kopmann, Merkel: Drei Jahre Telemac Open Source, 2013

den, sowie zwischen neun Advektionsverfahren und neun Gleichungslösern. Darüber hinaus können natürlich eigene Anpassungen programmiert werden. Dieser Vorteil birgt den Nachteil, dass bei so vielen Wahlmöglichkeiten nicht alle Kombinationen sinnvoll sind. Insbesondere für den Neueinsteiger sind die Einstellungen in den Validierungsbeispielen, sowie die Standardwerte hilfreich.

Die Entwicklungen für Telemac-2D sind im Vergleich zu den anderen Modulen weitgehend abgeschlossen und konsolidiert. Nichtsdestotrotz kommen immer noch Erweiterungen hinzu, wie die Berücksichtigungvon Regen und Evaporation (Version V6P2), die Parallelisierung der ThompsonRandbedingung (V6P1) und von Wehren (V6P2) oder die verbesserte Wandreibung für laterale Wände (V6P1). Ein nie abgeschlossener Entwicklungsbereich liegtin der Effizienz und Genauigkeit der Lösung. Eine neuere Entwicklung in diesem Bereich sind die Finite-Volumen-Verfahren (Zokagoa, Tchamen, HLLC und WAF, s. [3]). Vorteil dieser Verfahren ist neben der Massenkonservativität eine sehr gute Behandlung von Trockenfallen und Benetzen von Elementen sowie die Abbildung von Diskontinuitäten, wie sie unter anderem bei Dammbrüchen auftreten.

Für lokal begrenzte Modellgebiete und komplexe 3-D-Strömungen, oft im Nahfeld von Bauwerken, wird der Rechenaufwand eines 3-D-Modells wie Telemac-3D durch die deutlich bessere Abbildung der Strömung interessant. Der Umstieg von Telemac-2D auf Telemac-3D ist durch die Verwendung gleicher Dateiformate benutzerfreundlich. Bild 1 zeigt die tiefengemittelten Geschwindigkeiten für Validierungsbeispiel „221_Donau“, gerechnet mit Telemac-2D. Bild 2 zeigt vertikale Schnitte aus der Telemac-3D Berechnung mit 10 vertikalen Schichten. Die tiefengemittelten Fließgeschwindigkeiten unterscheiden sich nur geringfügig. Die Stromlinien und Profile zeigen anschaulich die detaillierteren Informationen aus einer 3-D-Berechnung und sollen den Mehrwert einer 3-DSimulation veranschaulichen.

Während Telemac-2D sich auch bei ungünstiger Wahl von Eingabeparametern als sehr stabil erweist, ist Telemac-3D empfindlicher und verlangt vom Benutzer mehr Fachwissen.

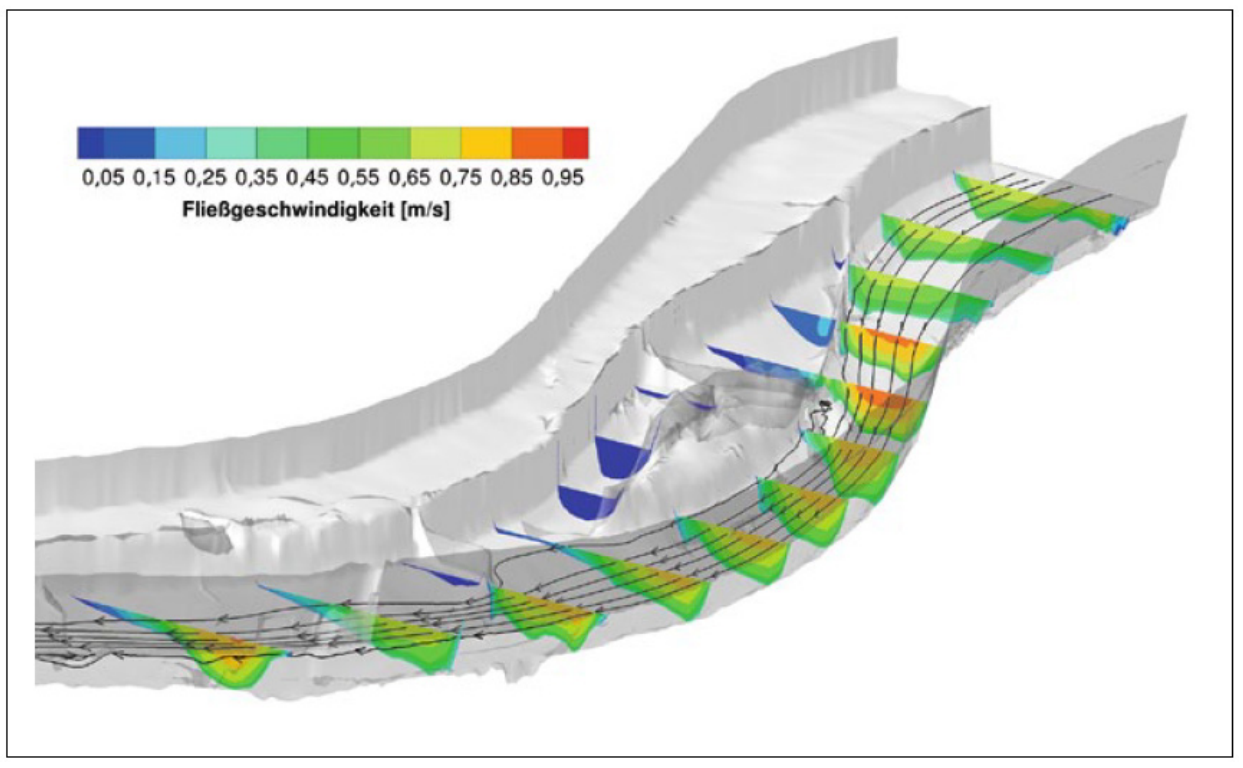




\section{Autorenfassung}

Kopmann, Merkel: Drei Jahre Telemac Open Source, 2013

Bild 2: $\quad$ Mit Telemac-3D berechnete Fließgeschwindigkeiten und Stromlinien für das Validierungsbeispiel „221_Donau“

\subsection{Sedimenttransport (Sisyphe)}

Egal ob in Ökologie, Energiewirtschaft, Verkehrswasserbau oder bei Standsicherheitsnachweisen, ohne Sedimenttransport lässt sich kaum eine mittelfristige prognosefähige Aussage über die Hydrodynamik in Fließgewässern treffen. Im täglichen Einsatz sind Untersuchungen zur Erosi-

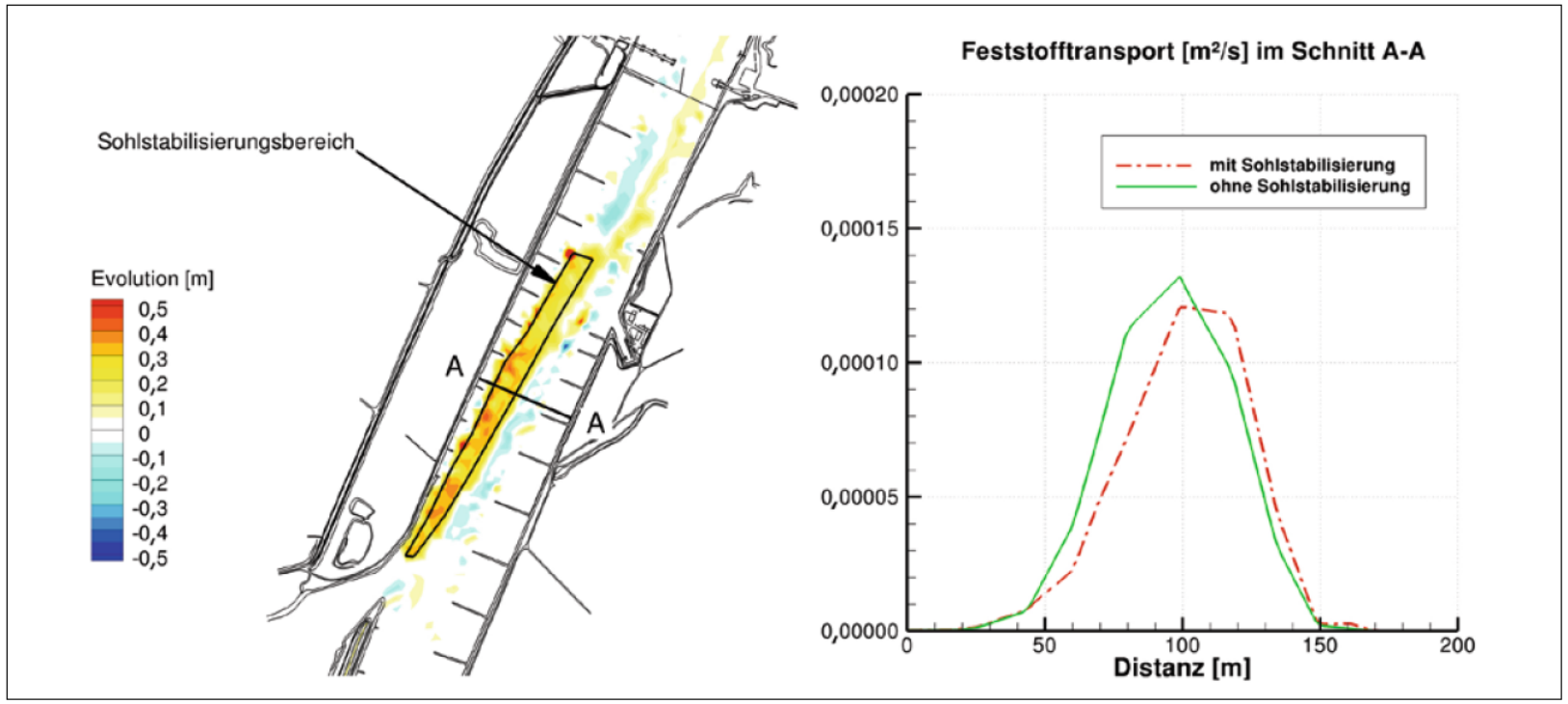

Bild 3: $\quad$ Sohlenänderungen nach einem Jahr infolge einer Sohlenstabilisierungsmaßnahme (links) und Feststofftransport bei bordvollem Abfluss mit und ohne Sohlenstabilisierung (rechts)

ons- und Böschungsstabilität, Evolutions-und Transportraten, Tracerverfolgung und stratigraphische Analysen bereits Standard [4], [5], [6].

Für fast alle morphodynamischen Prozesse sind in Sisyphe mehrere alternative Ansätze implementiert. Diese werden nicht nur vom Telemac-Konsortium, Universitäten und Drittentwicklern in deren Laborhallen entwickelt, sondern auch fortlaufend physikalisch validiert. Der Aufwand ist notwendig, weil viele der Ansätze im Sedimenttransport zum einen empirisch sind und zum anderen für mittlere Größen ermitteltwurden. Eine Verwendung dieser Ansätze in mehrdimensionalen 


\section{Autorenfassung}

Kopmann, Merkel: Drei Jahre Telemac Open Source, 2013

numerischen Simulationen bedarf sorgfältiger Anpassung und Validierung. Die Entwicklung neuer Ansätze unter Berücksichtigung einer hohen räumlichen und zeitlichen Auflösung profitiert durch die erhebliche Verbesserung der Messtechnik, erfordert aber auch einen enormen Aufwand.

Geschiebe- und Schwebstofftransport können mit Sisyphe unabhängig oder in direkter dynamischer Kopplung mit der Hydrodynamik berechnet werden.

Im Folgenden werden kurz drei Neuerungen vorgestellt, die seit der Quellcodefreigabe entstanden sind und nach Validierung seit kurzem auch der gesamten Telemac-Gemeinschaft zur Verfügung stehen.

\section{DredgeSim}

Zu den bemerkenswerten Neuentwicklungen gehört das Modul DredgeSim [7], das regelbasiert Baggerungen sowie Verklappungen im Gewässer simuliert und so die Auswirkungen anthropogener Maßnahmen auf den Sedimenthaushalt zeigt. Durch Vorgabe von Bagger- und Verklapppolygonen sowie Schwellenwerten für Fließtiefen lässt sich die Bewirtschaftungsstrategie an Fließgewässern abbilden und optimieren. Die BAW hat dieses Modul zum Einsatz an Bundeswasserstrassen am Institut für Wasserbau der Universität der Bundeswehr entwickeln lassen. Bild 3

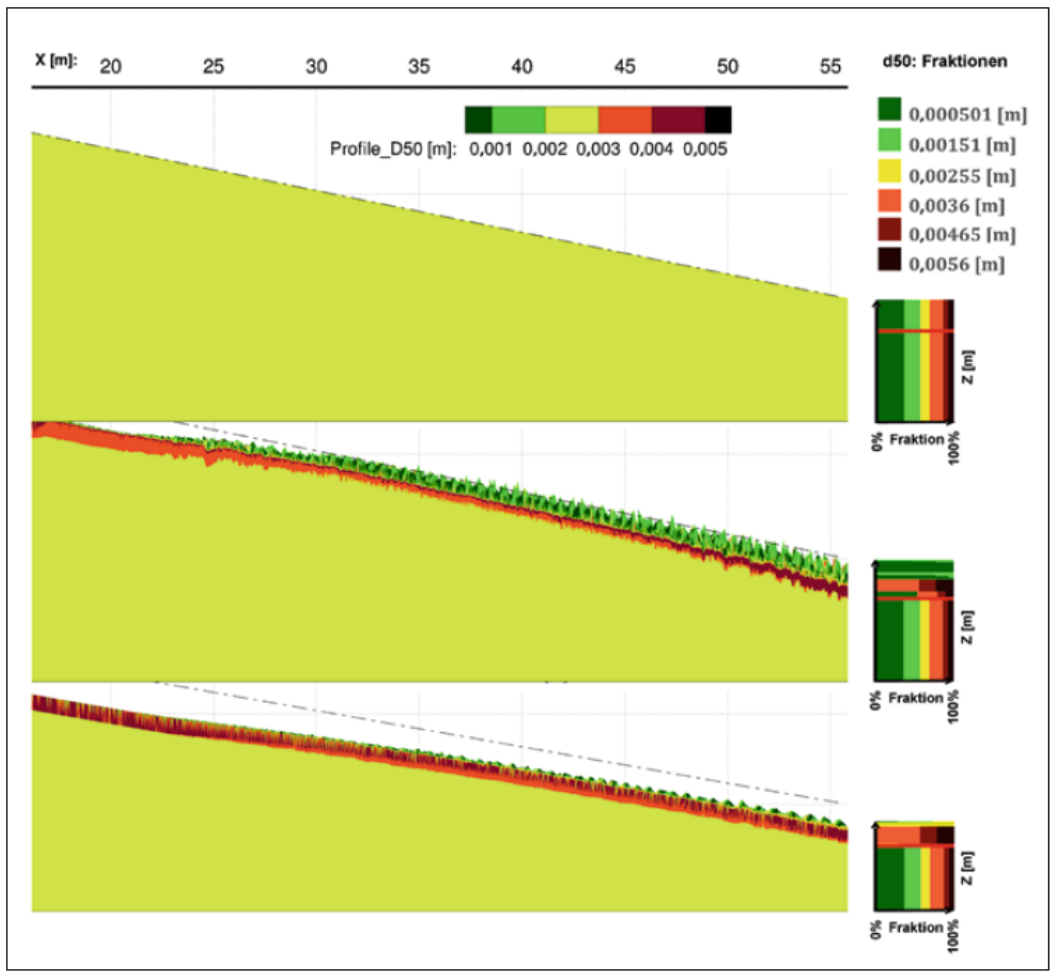

Bild 4: Zeitlicher Verlauf $(\mathrm{t}=0 \mathrm{~h} ; 6 \mathrm{~h} ; 24 \mathrm{~h})$ der simulierten Sohlenlagen für den Rinnenversuch von Günter [10] (Links mittlerer Durchmesser $\mathrm{d}_{50}$ im Längsschnitt; rechts: Anteile der Korngrößenklassen über die Tiefe (gemitteltes Profil)) 
zeigt beispielhaft die Auswirkung einer Sohlenstabilisierung auf die Sohlenänderung und Änderungen des Feststofftransports bei bordvollem Abfluss im Querschnitt.

\section{Kontinuierliches-Vertikales-Korngrößen-Sortiermodel (C-VSM)}

In Sisyphe wurde ein neues, optional zu verwendendes kontinuierliches Stratigraphiemodell „Continous Vertical Grain Sorting Model“ (C-VSM) implementiert [8], [9]. Durch eine möglichst genaue Abbildung der Korngrößenverteilung können die Kornrauheit und damit die Erosionsstabilität und die Ausprägung von Transportkörpern sowie eine mögliche spätere Aktivierung von Material besser simuliert werden. Das neue Modell trennt Buchhaltung und Simulation auf Basis einer adaptiven aktiven Schicht. So ist es möglich, bei Erosion und Sedimentation eine physikalisch unbegründete Durchmischung tieferer Schichten zu verhindern. Die Bearbeitung erfolgt auf Basis von kontinuierlichen Korngrößenverteilungsprofilen. Dies entspricht tiefenabhängigen Sieblinien von Bohrprofilen. Deren DiskretisierungmittelsPolylinien ist nur durch den Arbeitsspeicher limitiert.

Bild 4 zeigt die Anwendung des C-VSM auf die Modellierung des physikalischen Rinnenversuchs von Günter an der ETH Zürich [10]. Der Sedimentkörper ist als Längsschnitt dargestellt, die Einfärbung zeigt den mittleren Korndurchmesser. Über die Zeit entmischt sich das Sediment, das feinere (grüne) Material wird abtransportiert und das grobe (rote) Material bleibt als Deckschicht zurück. Die Erosion kommt zum Stillstand, wenn im Bereich der aktiven Schichtnur noch grobes unbewegliches Material übrig bleibt. Für dieselben Zeitschritte sind im tiefenabhängigen Siebliniendiagramm (räumlich gemittelt) die Anteile der einzelnen Korngrößen am Gemisch dargestellt.

\section{Einfaches Böschungserosionsmodel}

Der Kollaps instabiler Böschungen ist in Telemac und Sisyphe mit einem einfachen Algorithmus berücksichtigt und wurdeim Rahmen einer Doktorarbeit [11] weiterentwickelt. Wird durch morphologische Prozesse der Böschungswinkel zu steil, dann werden die betroffenen Knoten bis zum Erreichen des natürlichen Schüttwinkels verändert. Darüber hinaus wurde ein Übergang zu den bestehenden Nachbarknoten hergestellt, um Unstetigkeitsstellen im Netz zu vermeiden. Der zeitliche Verlauf dieser Anpassung ist vom Zeitschrittentkoppelt und wurde anhand von physikalischen Modellversuchen kalibriert sowie validiert. Das Model unterscheidet zwischen nassen und trockenen Bereichen sowie Materialvariationen.

\subsection{Wellenmodellierung (Artemis und Tomawac)}

Artemis (Agitation and Refraction with TElemac on a MIld Slope) und Tomawac (Telemac-based Operational Model Addressing Wave Action Computation) sind die Module zur Berechnung von Wellen innerhalb der Telemac-Suite. Während Artemis die elliptische Gleichung für geringe Nei- 
gungen, abgeleitet aus der Navier-Stokes-Gleichung unter verschiedenen Hypothesen, löst, berechnet Tomawac ein spektrales Seegangsmodell. Das Seegangspektrum wird durch Richtungen und Frequenzen beschrieben. Windenergieeintrag durch räumlich und zeitlich veränderliche Windfelder, Wellenbrechen, Reibung und der Energieaustausch zwischen Wellen sind einige der Funktionen.

\section{Paralleles Rechnen}

Bereits mit der seriellen Version von Telemac werden auf guten Bürocomputern Standardprobleme gelöst. Deutlich lässt sich der Anwendungsbereich der quelloffenen Software Telemac erweitern indem man mehrere Computergemeinsam einen Rechenlauf bearbeiten lässt. Gebietszerlegung ist die effizienteste und nützlichste Parallelisierung für FE-Programme. Dabei wird vollautomatisch das unregelmäßige Dreiecksnetz in Teilgebiete zerlegt. Jedes dieser Teilgebiete wird als komplett eigenständiges Rechenmodell auf verschiedenen Prozessoren oder sogar verschiedenen Computern berechnet, die an ihren Rändern Informationen austauschen. Bild 5 zeigt die Gebietszerlegung in 12 Gebiete für das offizielle Telemac-2D-Validierungsmodell „221_Donau“.

Die Schnittränder dieser Teilmodelle erhalten aberihre Randbedingungen (z. B. Fließgeschwindigkeiten und Wassertiefen)

nicht aus Benutzervorgaben, sondern für jedenZeitschritt über das Netzwerk von den Nachbargebieten (Programmieransatz: Message Passing Interface MPI). Gegenüber anderen Ansätzen, wie Multithreading und Vektorisierung, die nur mehrere CPU-Kerne auf einem Computer unterstützen, reduziert sich auch die Limitierung durch Arbeitsspeichertakt, Systembus und Festplattenzugriff. Durch die Gebietszerlegung werden zum einen weniger Daten pro Prozessor transferiert und zum anderen verfügt jed es Teilgebiet über einen eigenen Ein- und Ausgabekanal (In den meisten Fällen ist nicht die Prozessorleistung das schwächste Glied in der Berechnungskette, selbst wenn der schnellste Arbeitsspeicher zur Verfügung steht).

Diese Grundidee ist einfach und nicht nur großen Rechenzentren vorbehalten. Für kleinere Büros sind in etwa gleich schnelle Bürocomputer mit gewöhnlicher 1-GB-Ethernet-Netzwerke bereits effizient koppelbar, weil über das Netzwerk nur die Randbedingungen ausgetauscht werden. Beispielsweise genügen für Berechnungsgitter mit ca. 50000 Knoten 4 Bürocomputer à 4 Kernen für weniger als 500 Euro/Stück, um die Rechenzeit auf weniger als 1/10 gegenüber der klassischen seriellen Berechnung auf eben solchen Rechnern zu reduzieren und die Leistungsfähigkeit teuerer Cluster zu erreichen. Bild 5 zeigt die Skalierung der Rechenzeit für das Validierungsbeispiel „221_Donau“. 
Massive Parallelisierung mit teilweise einigen hundert Prozessoren wird an der BAW im täglichen Einsatz genutzt. Die bisher größten Modelle erreichten über 200 Mio. Knoten, verteilt auf 32768 Teilgebiete [12].

Wer nur projektbezogen kurzfristig sehr viele Varianten rechnen möchte, kann die Rechenleistung auf vorkonfigurierten Telemac-Clustern der University of Daresbury mieten. Noch mehr Rechenleistung vermieten Cloudcomputing-Anbieter wie Amazon EC2 und andere, hier ist aber eine eigene Systemeinrichtung nötig. Die Bezahlung erfolgt nach CPU-Kernen/h, somit lässt sich bereits vorab grob der finanzielle Aufwand schätzen. Diese Optionen sind insbesondere für kleine und mittlere Unternehmen attraktiv, die nicht permanent mit Telemac arbeiten oder sich die Computeradministration ersparen wollen.

\section{Die Benutzergemeinde}

Für den Telemac-Nutzer stehen eine Website (www.opentelemac.org) mit vielen Informationen und ein aktives Forum zur Verfügung. Im Forum sind sowohl die Entwickler des TelemacKonsortiums als auch Anwender und Entwickler aus zahlreichen Universitäten und Ingenieurbüros aktiv. Die Dokumentationen der verschiedenen Module werden in einem wiki-System kontinuierlich fortgeschrieben. Jährliche neue Versionen, Bugfixes und automatische Validierung an definierten Testbeispielen garantieren Kontinuität und eine effizienteWeiterentwicklung. Das CommunityPrinzip lebt vom Mitmachen. Darum werden explizit alle Nutzer gebeten, ihre Erfahrungen an die Entwickler zurückfließen zu lassen, selbst entwickelte Module in der Nutzergemeinschaft zur Diskussion zu stellen und Projekte vorzustellen. Ein gutes Forum dafür bietet auch die jährlich stattfindende TELEMAC-MASCARET User Conference (TUC), die 2013 zum 20. Geburtstag in Karlsruhe stattfand (www.baw.de/tuc2013). Zahlreiche Projektbeispiele in Form von Präsentationen und Proceedings der TUC-Teilnehmer seit 2005 findet man unter www.opentelemac.org/index.php/user-conference.

\section{Zukünftige Entwicklungslinien}

Die Anzahl der Entwickler steigt und auch in Zukunft wird es zahlreiche Neuerungen geben, um die Genauigkeit zu verbessern, die Geschwindigkeit zu erhöhen und die Funktionalität zu erweitern. An zahlreichen Teilprojekten wird bereits seit Jahren gearbeitet. Dazu gehören folgende Punkte:

\section{Generelles}

- Einbinden von weiteren Modulen für Spezialanwendungen, wie das Modul zur Berechnung von Algenausbreitung und ein Modul zur Berechnung von Ölkatastrophen unter Berücksichtigung von Wind, Wellen, Tiden. Entwicklung und Validierung an physikalischen Modellen ist bereits seit einigen Jahren im Gange (EDF).

- Nutzerschnittstellen für Salome von der EDF und Pyxis von HR Wallingford.

- Vereinfachung des Installationsvorgangs. 


\section{Autorenfassung}

Kopmann, Merkel: Drei Jahre Telemac Open Source, 2013

- Telemac-2D soll ersetzt werden durch ein 1-Layer-Telemac3D, um die Entwicklungslinien zusammenzuführen und Neuerungen immer sofort für beide Verfahren verfügbar zu haben.

- Werkzeuge zur Berechnung von Sensitivitäten, Unsicherheiten und Parameterschätzungen [13].

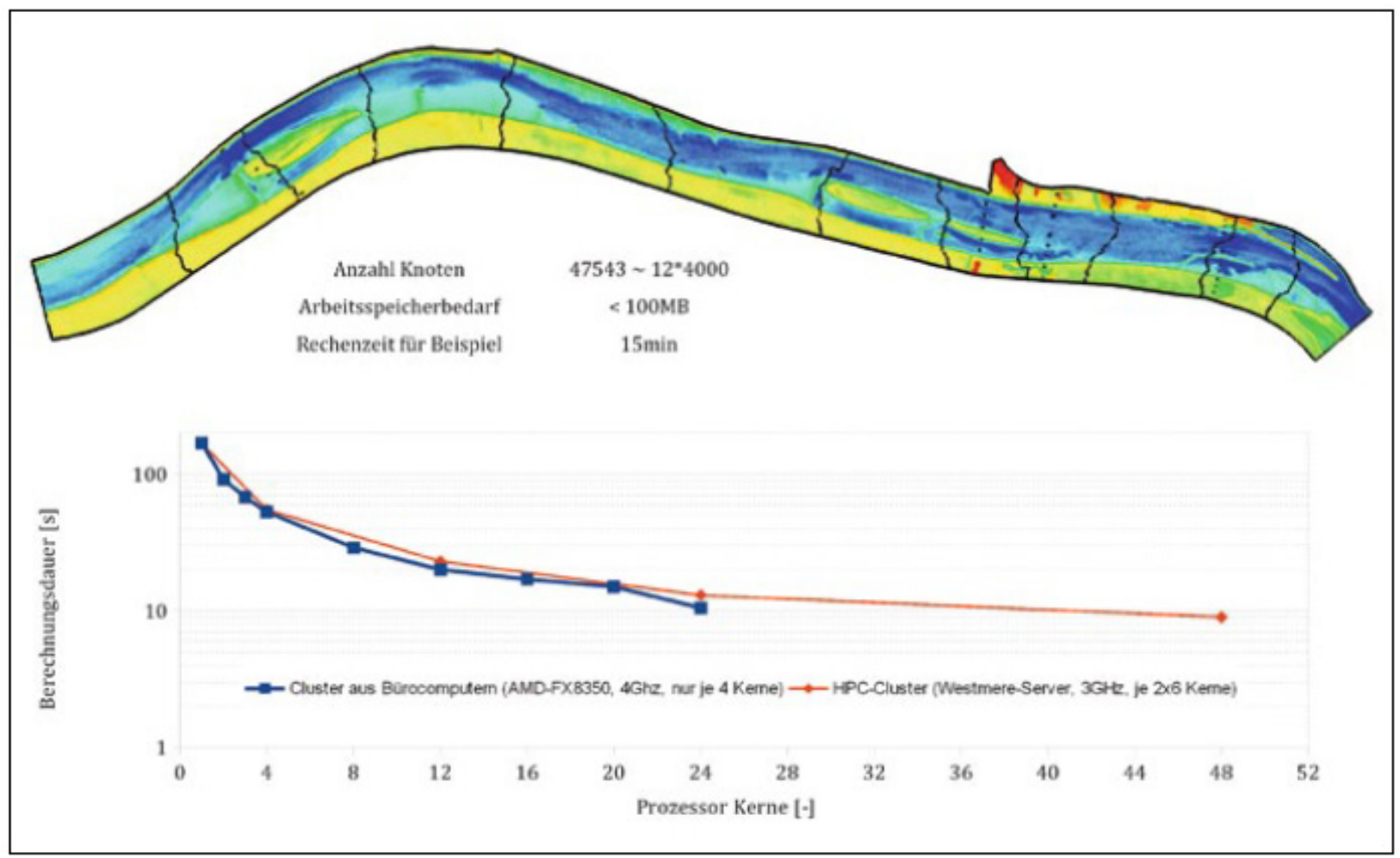

Bild 5: Berechnungsdauer Vergleich am Validierungsmodel „221_Donau“ automatisch zerlegt in 12 Teilmodelle mit parallelem Bürocomputer mit AMD-FX8350-Prozessoren und HPC-Server-Cluster SGI Altix ICE 8400

\section{Numerik}

- Verbesserung der Berechnungsgenauigkeit durch deutlich verfeinerte Advektionsverfahren unter Berücksichtigung von trockenfallenden Knoten mit Hilfe von semi-impliziten, unkonditionierten, stabilen, konservativen, mo-

noton und distributiv verteilten Schemata.

- Erreichung der binären Gleichheit zwischen seriellen und parallelen Ergebnissen. 


\section{Autorenfassung}

Kopmann, Merkel: Drei Jahre Telemac Open Source, 2013

- Langzeitentwicklung: Aufbau eines auch in der Vertikalen unstrukturierten 3-D-Modells, damit im Netz Düsen in Wänden oder Hindernisse direkt eingebettet werden können.

\section{Verbesserung einzelner Module:}

- Mehrkornansatz in Telemac-3D.

- Vereinheitlichung von kohesiven, nicht-kohesiven und gemischten Sedimenten in Sisyphe.

- Optimierung von Tomawac.

\section{Zusammenfassung}

Seit der Veröffentlichung des Quellcodes der Telemac-Suite entwickelt sich das Programm schneller denn je zuvor. Die Beiträge der Nutzergemeinde führen zu bedeutenden Verbesserungen und zur permanenten Validierung. Qualität und Kontinuität werden mit erheblichem personellem und finanziellem Aufwand vom Telemac-Konsortium sichergestellt. Die hier kurz angerissenen Möglichkeiten und die Projektbeispiele in diesem Themenheft der WasserWirtschaft sollen potentielle Anwender dazu animieren, die Telemac- Suite zu testen, nutzen und durch Rückmeldungen oder Softwareentwicklung beizutragen. Die Autoren sprechen daher besonders auch kleinere Ingenieurbüros, Behörden oder einzelne Forscher an, für die quelloffene Software auch von finanzieller Bedeutung ist.

\section{Danksagung}

Die Autoren danken Jean-Michel Hervouet für die Informationen zu den Entwicklungslinien der Telemac-Suite und seine Unterstützung für dieses Themenheft.

\section{Autoren}

\section{Dr.-Ing. Rebekka Kopmann}

Bundesanstalt für Wasserbau

Kußmaulstr. 17

76167 Karlsruhe

Rebekka.kopmann@baw.de

\section{Dr.-Ing. Uwe H. Merkel}

Ingenieurbüro Merkel

Hausackerstraße 9

76185 Karlsruhe

uwe.merkel@uwe-merkel.com 


\section{Literatur}

[1] Goll, A. Kopmann, R. Kontinuierliche Validierung hydrodynamischer und morphodynamischer Modelle mit physikalischen Modellversuchen. In: WasserWirtschaft 103 (2013), Heft 12, S. 28-33.

[2] Villaret, C.; Hervouet, J. M.; Merkel, U.; Kopmann, R.: Morphodynamic modelling using the Telemac finite-element System. In: Computers and Geosciences 53 (2013), S. 105-113.

[3] Ata, R.: TELEMAC-2D new finite volume schemes for shallow water equations with source terms on 2D unstructured grids. In: Proceedings of the XIXth TELEMAC-MASCARET User Conference. (2012) (www.opentelemac.org).

[4] Wurms, S.; Schröder, P. M.: 2-D-morphodynamische Simulationen zur Anpassung des Verkehrswasserbaus an den Klimawandel. In: WasserWirtschaft 103 (2013), Heft 12, S. 16-21.

[5] Dorfmann, C.; Zemz, G.: Numerische Untersuchungen mit Telemac bei Wasserkraftanlagen zwei Fallstudien. In: WasserWirtschaft 103 (2013), Heft 12, S. 41-46.

[6] Putzar, B.: Simulation natürlicher und anthropogener Prozesse im Küstenraum mit der TelemacSuite. In: WasserWirtschaft 103 (2013), Heft 12, S. 34-40.

[7] Maerker, C.; Malcherek, A.: Die Analyse von Baggern und Verklappen-Teil 1: Das Softwarepaket DREDGESIM. In: KW Korrespondenz Wasserwirtschaft 3 (2010), Nr. 10, S. 550-555.

[8] Merkel, U.; Kopmann, R.: Continuous Vertical Grain Sorting for Telemac \& Sisyphe v6p2, In: Proceedings of the XIXth TELEMAC-MASCARET User Conference. (2012). (www.opentelemac.org).

[9] Merkel, U.; Kopmann, R. A.: Continuous Vertical Grain Sorting Model for Telemac \& Sisyphe. In: River Flow 2012. CRC Press, 2012.

[10] Günter, A.: Die kritische mittlere Sohlenschubspannung bei Geschiebemischungen unter Berücksichtigung der Deckschichtbildung und der turbulenzbedingten Sohlenschubspannungsschwankungen. In: Mitteilungen der VAW der ETH Zürich (1971), Nr. 3.

[11] Die Moran, A.: Physical and numerical modelling investigation of induced bank erosion as a sediment transport restoration strategy for trained rivers: the case of the Old Rhine (France). Thèse Université Paris-Est, 2012 (http://research.edf.com/fichiers/fckeditor/ Commun/Innovation/theses/TheseDieMoran. pdf; Aufruf: 15.09.2013). 


\section{Autorenfassung}

Kopmann, Merkel: Drei Jahre Telemac Open Source, 2013

[12] Moulinec, C.; Audouin, Y.; Sunderland, A.: Optimizing TELEMAC-2D for Large-scale Flood Simulations. 2011 (www.prace-ri.eu/IMG/pdf/ Optimizing_TELEMAC-2D_for_Large-scale_ Flood_Simulations.pdf; Aufruf: 15.09.2013).

[13] Merkel, U.; Riehme, J.; Naumann, U.: Rückrechnung von Rand- und Anfangsbedingungen mit Telemac und Algorithmischer Differentiation. In: WasserWirtschaft 103 (2013), Heft 12, S. 22-27.

Rebekka Kopmann and Uwe H. Merkel

\section{Three Years of Telemac Open Source}

The paradigm shift in hydrodynamic modelling software licensing changed our business. Moving away from black box to open source code, developed by a community and in terms of quality and consistency supervised by the Telemac consortium, started a dynamic development of new ideas and features. This article and the full Telemac special issue aims to animate engineers in private companies as well as research engineers to test and use the Telemac-Suiteand to participate in the community.

Ребекка Копманн и Уве Х. Меркель

\section{Открытому программному обеспечению Telemac Open Source 3 года}

Изменение парадигм цифрового программного обеспечения для гидравлики проточных водоемов существенно изменило отрасль. С переходом к открытым, общественно базированным разработкам крупных пакетов программного обеспечения, таких как Теlemac-Suite, возникает усиленная динамика: количество пользователей значительно возрастает и вместе с этим возникает много новых идей, разработок и проектов. Консорциум Telemac координирует и контролирует такие аспекты как континуальность и качество. В статье описывается, насколько данное программное обеспечение может стать полезным для работы инженеров плановых бюро и государственных учреждений, а также при проведении научных исследований. 